\title{
Division of Cancer Prevention
}

National Cancer Institute

\section{Source}

National Cancer Institute. Division of Cancer Prevention. NCI Thesaurus. Code C18750.

Reorg anized on October 1, 1997 to the extramural Division of Cancer Prevention and the extramural Division of Cancer Control and Population Science. 